\title{
Direkte Demokratie, Steuermoral und Steuerhinterziehung: Erfahrungen aus der Schweiz
}

\author{
Gebhard Kirchgässner*
}

(Universität St. Gallen, Schweizerisches Institut für Außenwirtschaft und Angewandte Wirtschaftsforschung, CESifo und Leopoldina)

\section{Einleitung}

Die Frage, welchen Einfluss die direkte Demokratie auf die Steuermoral hat, könnte im Prinzip sehr schnell beantwortet werden, indem erstens festgestellt wird, dass es einen solchen Einfluss gibt, und zweitens, dass er positiv ist: Je mehr direkte Volksrechte den Bürgerinnen und Bürgern zur Verfügung stehen, desto besser ist die Steuermoral. Dies wurde gerade eben wieder in einer Arbeit von Torgler (2005) nachgewiesen. Und dass dies quantitativ durchaus von Bedeutung ist, haben bereits Weck-Hannemann und Pommerehne (1989) aufgezeigt. Damit, bzw. mit einer kurzen Beschreibung der Ergebnisse dieser Arbeiten, könnte man es eigentlich belassen.

Wenn die direkte Demokratie der Steuermoral förderlich ist, dann sollte die Steuermoral in der Schweiz besonders hoch sein. Schließlich ist die Schweiz der einzige Staat auf der Welt, der auch auf der nationalen Ebene stark ausgeprägte direkte Volksrechte kennt. Glaubt man den internationalen Umfragen, dann ist dies jedoch zumindest heute nicht mehr der Fall: Die Schweizer haben im Gegenteil eine eher schlechte Steuermoral. In der Umfrage des International Social Survey Programme (ISSP) aus dem Jahr 1998 liegt die Schweiz von 32 berücksichtigten Staaten auf Platz 24, und gemäß den Angaben des World Value Survey (WVS) liegt sie im Durchschnitt der Jahre 1999 bis 2002 auf dem 60sten von 80 Plätzen. ${ }^{1}$ Dabei ist die Steuermoral seit Beginn der 1990er Jahre

\footnotetext{
*Prof. Dr. Gebhard Kirchgässner, Universität St. Gallen, SIAW-HSG, Bodanstrasse 8, CH-9000 St. Gallen, Schweiz. Email: Gebhard.Kirchgaessner@unisg.ch. Vortrag im Rahmen des 27. Universitätsseminars „Dialog Wissenschaft und Praxis“, Schloss Gracht in Erfstadt-Liblar, 3.-5. Juni 2005. Überarbeitete Fassung, September 2006. Ich danke einem anonymen Gutachter für hilfreiche Hinweise.
}

1. Siehe hierzu die im Anhang in Tabelle A1 wiedergegebenen Werte. Die Werte des ISSP finden sich 


\section{Direkte Demokratie, Steuermoral und Steuerhinterziehung}

deutlich schlechter geworden: Im Durchschnitt der Jahre 1989 und 1990 lag die Schweiz von den 18 in Torgler (2004) aufgeführten europäischen Ländern immerhin auf Platz 4. Diese Verschlechterung in den Plätzen zeigt auch, dass die Steuermoral in der Schweiz nicht nur absolut abgenommen hat, sondern auch im Vergleich zu den anderen Ländern. Zumindest auf den ersten Blick scheinen die aktuellen Umfrageergebnisse in krassem Gegensatz zu den Ergebnissen von Torgler (2005) oder Weck-Hannemann und Pommerehne (1989) zu stehen. $^{2}$

Nun könnte man einwenden, dass diese in Umfragen erhobenen Zahlen unzuverlässig sind, da die Antworten auf die hier gestellten Fragen kulturabhängig sind und deshalb über die Länder hinweg kaum verglichen werden können. Auch wenn dieser Einwand gegen praktisch alle der in den letzten Jahren veröffentlichten Arbeiten vorgebracht werden kann, die mit Daten dieser oder ähnlicher Surveys arbeiten, kann die Möglichkeit einer Kulturabhängigkeit der Antworten nicht bestritten werden; schließlich konnte bei Umfragen nach der persönlichen Zufriedenheit bzw. dem "Glück“ der Individuen ein erheblicher kultureller Einfluss aufgezeigt werden. ${ }^{3}$ Andererseits ergeben sich auch dann, wenn wir die Stichprobe auf westlich orientierte Länder wie die OECD einschränken, für die Schweiz keine besseren Ergebnisse: Nach den LVS-Daten liegt sie auf Platz 19 von 24 Ländern, nach den ISSP-Daten auf Platz 15 von 21 erfassten Ländern. ${ }^{4}$

Aber auch wenn man kaum bestreiten kann, dass die - so gemessene - Steuermoral in der Schweiz niedrig ist, muss zwischen diesen Ergebnissen und der Behauptung, dass stärker ausgebaute Volksrechte - ceteris paribus - zu geringerer Steuerhinterziehung führen, kein Widerspruch bestehen. Die Steuermoral in der Schweiz könnte ohne die direkten Volksrechte ja noch geringer sein. Zudem gibt es, worauf unten eingegangen wird, zumindest zwei plausible Argumente dafür, warum die Schweizer Bevölkerung Steuerhinterziehung eher toleriert als die Bevölkerungen vieler anderer Staaten. Auch muss eine geringe Steuermoral noch nicht bedeuten, dass viel hinterzogen wird. Es könnte ja sein, dass die direkten Volksrechte dafür sorgen, dass trotz einer geringen

unter http://www.za.uni-koeln.de/data/en/issp/codebooks/s3190cdb.pdf, S. 133 (Frage V16 für die Steuermoral und Frage V22 für das Vertrauen in das Rechtssystem). Zum World Value Survey siehe Inglehart et al. (2004) (Frage E085 für das Vertrauen in das Rechtssystem und Frage F116 für die Steuermoral).

2. Die früheren Umfrageergebnisse sind auch noch eher im Einklang mit den Daten, die Weck (1983) von Tretter (1974) übernommen und in ihren Schätzungen zur Bestimmung der Größe der Schattenwirtschaft verwendet hat. Danach hat die Schweiz von allen von ihr betrachteten Ländern die beste Steuermoral.

3. Siehe hierzu Dorn et al. (2006).

4. Neben den Ländern in Westeuropa und Nordamerika berücksichtigen wir hier Israel, Australien und Neuseeland. Auch die Tatsache, dass die WVS-Daten für die Schweiz (und Australien) auf dem Durchschnitt der Jahre 1995 bis 1997 beruhen, für die anderen Länder aber auf dem Durchschnitt der Jahre 1999 bis 2001, dürfte am Ergebnis kaum etwas ändern. Schließlich führen für die Schweiz beide Umfragen zu einem ähnlich gleichen schlechten Ergebnis. 


\section{Gebhard Kirchgässner}

Steuermoral die Steuergesetze relativ gut befolgt werden. Dafür aber ist es notwendig, zum einen den Zusammenhang zwischen direkten Volksrechten und Steuermoral sowie zweitens den Zusammenhang zwischen direkten Volksrechten und Steuerhinterziehung genauer zu betrachten. Dabei soll als gegeben angenommen werden, dass eine bessere Steuermoral - ceteris paribus - zu einer höheren Steuerehrlichkeit führt. Entsprechende Untersuchungen liegen für die Schweiz leider nicht vor und sind auch - angesichts der Datenlage zumindest derzeit kaum durchzuführen. ${ }^{5}$

Neben der Steuermoral sollte auch das Vertrauen in die politischen Institutionen eine wesentliche Rolle für die Bereitschaft, Steuern zu zahlen, spielen. Wenn die Bürgerinnen und Bürger diesbezüglich ein hohes Vertrauen haben, können sie davon ausgehen, dass ihre Steuergelder nicht verschleudert werden. Auch dies sollte sowohl die Steuermoral als auch die Bereitschaft, Steuern zu zahlen, fördern. Betrachtet man das Vertrauen in das Rechtssystem, welches in beiden Surveys ebenfalls abgefragt wird, dann liegt die Schweiz bei den WVS-Daten auf Platz 8 von 46 und beim ISSP-Survey auf Platz 10 von 32 untersuchten Staaten. ${ }^{6}$ Beschränkt man die Stichprobe wieder auf die westlich orientierten Staaten, so liegt die Schweiz bei beiden Datensätzen auf Platz 8 von jeweils 22 berücksichtigten Ländern. Dies sind immerhin Plätze im Mittelfeld; das Vertrauen der Schweizerinnen und Schweizer in ihr Rechtssystem scheint zumindest stärker ausgeprägt (oder weniger stark angeschlagen) zu sein als ihre Steuermoral.

Bevor auf die Fragen eingegangen werden kann, welchen Einfluss Steuermoral und Vertrauen auf das Verhalten der Steuerzahler haben, muss zunächst geklärt werden, was "Vertrauen“ und "Steuermoral“ genau bedeuten und wie sie gemessen werden. Dies soll im folgenden Abschnitt 2 geschehen. Bezüglich der Steuermoral herrscht gerade im deutschen Sprachraum häufig ein Missverständnis vor, indem sie mit Steuerehrlichkeit (weitgehend) gleichgesetzt wird. Tatsächlich aber handelt es sich zum einen um eine moralische Einschätzung und zum anderen um konkretes Handeln, und beides muss nicht notwendigerweise übereinstimmen. In Abschnitt 3 wird aufgezeigt, in welcher

5. Experimentelle Evidenz dazu liefern Bosco und Mittone (1997). Darüber hinaus präsentiert Weck (1983) empirische Evidenz dafür, dass eine bessere Steuermoral zu geringerer Schattenwirtschaft führt. Da eine Tätigkeit in der Schattenwirtschaft automatisch Steuerhinterziehung beinhaltet, sollte deshalb eine bessere Steuermoral auch zu geringerer Steuerhinterziehung führen. Dass Steuerehrlichkeit etwas mit Moral zu tun haben muss, ergibt sich im Übrigen auch daraus, dass es angesichts der geringen Aufdeckungswahrscheinlichkeit und Strafen für die Bürgerinnen und Bürger rational wäre, Steuern zu hinterziehen. Siehe hierzu Alm, McClelland und Schulze (1992) sowie Andreoni, Erard und Feinstein (1998). Wenn man nicht auf extreme Risikoaversion zurückgreifen will, kann die Tatsache, dass die Mehrheit der Bürgerinnen und Bürger keine oder zumindest kaum Steuern hinterzieht, kaum anders als mit moralischen Beweggründen erklärt werden.

6. Es gibt im ISSP-Survey keine Frage für das allgemeine Vertrauen in die politischen Institutionen. Die Frage nach dem Vertrauen in das Rechts- und Gerichtswesen dürfte von den gestellten Fragen noch am ehesten in diese Richtung gehen. Daher verwenden wir für beide Surveys diese Frage. 


\section{Direkte Demokratie, Steuermoral und Steuerhinterziehung}

Weise die direkten Volksrechte, so wie sie in der Schweiz verwirklicht sind, den Bürgerinnen und Bürgern die Möglichkeit eröffnen, auf die Besteuerung einzuwirken. Danach befassen wir uns mit den Auswirkungen der direkten Demokratie auf die Steuermoral (Abschnitt 4), auf die Steuerhinterziehung (Abschnitt 5) sowie auf das Verhältnis zwischen den Steuerbehörden und den Bürgern (Abschnitt 6).

\section{Bedeutung und Erfassung von Steuermoral und Vertrauen}

Unter Steuermoral wird im Deutschen häufig das Ausmaß verstanden, in welchem die Bürgerinnen und Bürger ihrer „Pflicht“ , Steuern zu zahlen, nachkommen. Genau betrachtet unterscheidet sich diese „Befolgung der Steuergesetze“ aber von der eigentlichen Steuermoral. Im Englischen wird dies deutlicher: dort wird zwischen "tax compliance" und „tax morale" unterschieden. Die Steuermoral (im engeren Sinn) betrifft dann die Frage, in welchem Ausmaß es die Bürgerinnen und Bürger als ihre moralische Pflicht ansehen, ihre Steuern zu zahlen. Dass es sich hier um eine moralische Pflicht handelt, setzt voraus, dass es über die legalen Bestimmungen hinaus gerechtfertigte Gründe dafür gibt, dass die Steuern entrichtet werden. Wie diese Gründe aussehen, d.h. unter welchen Bedingungen die Bezahlung von Steuern als moralische Pflicht der Bürgerinnen und Bürger betrachtet werden kann, ist seit langem nicht nur ein Problem der Wirtschaftswissenschaft, sondern vor allem der Philosophie und auch der Theologie. ${ }^{7}$ Bei beidem, der Steuerehrlichkeit wie auch der Steuermoral, dürfte auch die Struktur des Steuersystems eine wesentliche Rolle spielen. ${ }^{8}$

Die Steuermoral kann daher (und wird vermutlich auch) einen Einfluss auf die Befolgung der Steuergesetze haben, aber sie kann nicht damit gleichgesetzt werden. Sie kann mit Hilfe von Befragungen erfasst werden. Im International Social Science Panel geschieht dies sehr direkt mit folgender Fragestellung:

Um weniger Steuern zahlen zu müssen, gibt ein Steuerzahler nicht sein gesamtes Einkommen an. Betrachten Sie dies als (i) nicht unrechtmäßig, (ii) ein bisschen unrechtmäßig, (iii) unrechtmäßig, oder (iv) schwer unrechtmäßig?9

Ordnet man den einzelnen Antworten Werte von 1 bis $4 \mathrm{zu}$, wobei „1“ die höchste und "4" die geringste Steuermoral abbildet, und bildet man gewichtete Mittelwerte, dann ergibt sich daraus ein Index, dessen Wertebereich zwischen 1 und 4 liegt. ${ }^{10}$ Dessen Mittelwert liegt bei 2,07 mit einer Standardabweichung

7. Siehe hierzu auch Kirchgässner (2003, S. 221ff.).

8. Siehe hierzu z.B. Feld und Tyran (2002) sowie Vihanto (2003).

9. Die genauen Kategorien sind: (i) not wrong, (ii) a bit wrong, (iii) wrong, (iv) seriously wrong.

10. Die hier betrachteten Werte sind nationale Mittelwerte. Die gesamte Stichprobe umfasst bei den ISSP-Daten 39.034 Personen, im WVS 89.678 Personen. 


\section{Gebhard Kirchgässner}

von 0,24. Der Wert für die Schweiz liegt mit 2,23 deutlich über dem Mittelwert; wie bereits erwähnt liegt die Schweiz damit auf Platz 24 von 32 in der Stichprobe enthaltenen Ländern. In Ostdeutschland ist die Steuermoral fast genauso gut wie in der Schweiz, mit einem Wert von 2,24 kommt es auf den 25. Platz. Dagegen kommen Westdeutschland mit einem Wert von 2,43 auf den drittletzten und Österreich mit einem Wert von 2,52 sogar nur auf den vorletzten Platz. Insgesamt scheint die Steuermoral in den deutschsprachigen Ländern sehr gering zu sein, wobei sie in der Schweiz noch am höchsten ist.

Die (sinngemäße) Fragestellung im World Value Survey ist leicht anders:

Glauben Sie, dass es immer, niemals, oder gelegentlich gerechtfertigt werden kann, Steuern zu hinterziehen, wenn sich die Gelegenheit dazu bietet?

Dabei wird den Befragten eine 10 Punkte-Skala vorgelegt, wobei „1“ bedeutet, dass Steuerhinterziehung nie, und „10“, dass sie immer gerechtfertigt werden kann. Wie bei den ISSP-Daten kann man daraus einen Index bilden. Sein Mittelwert liegt bei 2,31 mit einer Standardabweichung von 0,71. Die Schweiz hat einen Wert von 2,65 und kommt damit auf den 60. Platz. Deutschland erhält mit einem Wert von 2,37 den 53. Platz, und Österreich mit einem Wert von 2,10 sogar den 35 . von 80 betrachteten Ländern. Für die Schweiz ist damit das Ergebnis kaum besser als mit den ISSP-Daten.

Betrachtet man die Daten genauer, fällt auf, dass die Korrelation für die 28 Staaten, die in beiden Stichproben enthalten sind, mit 0,513 doch etwas gering ist. Dies lässt Zweifel an der Aussagekraft der Daten aufkommen. Da die Schweiz jedoch in beiden Stichproben relativ schlecht abschneidet, spricht vieles dafür, dass die Steuermoral in der Schweiz - im internationalen Vergleich - tatsächlich recht gering ist.

Das Vertrauen in das Rechts- und Gerichtswesen wird im ISSP-Datensatz (sinngemäß) mit folgender Fragestellung erfasst:

Allgemein gesprochen, würden Sie sagen, dass man Vertrauen in das Rechts- und Gerichtssystem haben kann? Kann man (i) vollständiges Vertrauen, (ii) großes Vertrauen, (iii) etwas Vertrauen, (iv) sehr wenig Vertrauen bzw. (v) gar kein Vertrauen haben?

Ordnet man den einzelnen Antworten Werte von 1 bis $5 \mathrm{zu}$, wobei „1“ das stärkste und „5“ die Abwesenheit jeglichen Vertrauens bedeutet, dann ergibt sich ein Index, dessen Wertebereich zwischen 1 und 5 liegt, mit einem Mittelwert von 3,84 und einer Standardabweichung von 0,92. Der Wert für die Schweiz liegt mit 3,43 etwas unter dem Mittelwert; wie bereits erwähnt liegt die Schweiz damit auf Platz 10 der 32 betrachteten Länder. In Westdeutschland ist das Vertrauen mit einem Indexwert von 3,38 und Platz 9 geringfügig besser, in Ostdeutschland mit einem Indexwert von 4,29 und dem Rang 19 dagegen massiv schlechter. Am besten von allen deutschsprachigen Ländern ist das Vertrauen in das Rechts- und Gerichtswesen in Österreich ausgeprägt; mit einem Indexwert von 2.62 liegt es auf Platz 5. 


\section{Direkte Demokratie, Steuermoral und Steuerhinterziehung}

Beim WVS-Panel ist die Fragestellung (wiederum sinngemäß) sehr ähnlich:

Wie viel Vertrauen haben Sie in das Rechtssystem: (i) sehr viel, (ii) ziemlich viel, (iii) nicht sehr viel oder (iv) überhaupt kein Vertrauen?

Ordnet man den einzelnen Antworten Werte von 1 bis 4 zu, wobei „1“ wieder das stärkste und „4" die Abwesenheit jeglichen Vertrauens bedeutet, dann liegt der Mittelwert des daraus gebildeten Index bei 2,61 mit einer Standardabweichung von 0,24. Der Wert für die Schweiz liegt mit 2,31 deutlich unter dem Mittelwert; wie bereits erwähnt liegt die Schweiz damit auf Platz 6 der 46 betrachteten Länder. In Deutschland ist das Vertrauen mit einem Indexwert von 2,35 und Platz 7 geringfügig schlechter. Am besten von allen deutschsprachigen Ländern ist das Vertrauen in das Rechts- und Gerichtswesen in Österreich ausgeprägt; mit einem Indexwert von 2,19 liegt es auf Platz 3. Im Gegensatz zur Steuermoral ist hier die Übereinstimmung der beiden Umfragen deutlich höher; die Korrelation zwischen den beiden Indexreihen beträgt 0,707.

Man sollte annehmen, dass die Steuermoral und das Vertrauen in das Rechtsund Gerichtswesen miteinander (hoch) korreliert sind; tatsächlich aber scheinen sie voneinander weitgehend unabhängig zu sein: Die Korrelation zwischen den Indexwerten beträgt beim WVS 0,210 und beim ISSP sogar nur 0,009. Dass die Reihen kaum miteinander korreliert sein können, wird deutlich, wenn man einzelne Länder betrachtet. Im ISSP-Datensatz liegt Österreich bei der Steuermoral auf dem zweitletzten Platz, beim Vertrauen dagegen auf Platz 5. Umgekehrt liegt Japan bei der Steuermoral auf Platz 1, beim Vertrauen dagegen nur auf Platz 16. Dänemark liegt dagegen (wie auch die anderen skandinavischen Staaten) in beiden Skalen weit vorne: bei der Steuermoral auf Platz 6 und beim Vertrauen sogar auf Platz 1.

Gründe dafür, dass in der Schweiz die Steuermoral nicht nur heute relativ gering ist, sondern auch in den neunziger Jahren deutlich abgenommen hat, können zum einen die Diskussion über eine Steueramnestie sein, die in der ersten Hälfte der neunziger Jahre stattgefunden hat, und zum anderen die öffentlich immer wieder vorgebrachte Verteidigung des Bankgeheimnisses. Eine Steueramnestie können ehrliche Steuerzahler als ungerecht empfinden, da dabei implizit diejenigen belohnt werden, die nicht ehrlich waren. Dies kann sich negativ auf die Steuermoral auswirken.

Bezüglich des Bankgeheimnisses kennt die Schweiz bekanntlich die weltweit wohl einzigartige Unterscheidung zwischen Steuerhinterziehung und Steuerbetrug. Das falsche Ausfüllen einer Steuererklärung allein ist auch dann, wenn es mit voller Absicht geschieht und wenn erhebliche Summen im Spiel sind, kein straffähiges Delikt, es wird nur dann dazu, wenn es in Zusammenhang mit einer Urkundenfälschung geschieht. Dann aber spricht man nicht mehr von Steuerhinterziehung, sondern von Steuerbetrug.

Diese Unterscheidung ist die Grundlage des schweizerischen Bankgeheimnisses: Die Schweiz leistet Rechtshilfe nur bei Steuerbetrug, nicht aber bei Steuerhinterziehung. Damit wird die Steuerhinterziehung faktisch als Kavaliersdelikt behandelt. Dies spiegelt sich in den Einschätzungen der moralischen 


\section{Gebhard Kirchgässner}

Qualifikation der Steuerhinterziehung durch die Bevölkerung in der Schweiz wider. Insofern sind die Umfrageergebnisse nicht erstaunlich. Auch der Rückgang der Steuermoral seit Beginn der neunziger Jahre wird verständlich, wenn man berücksichtigt, dass das Bankgeheimnis, das heute freilich auch in der Schweiz nicht mehr unumstritten ist, ${ }^{11}$ seit damals international immer stärker unter Druck geraten ist und in der Schweiz entsprechend vehement verteidigt wurde. ${ }^{12}$ Man kann die hier faktisch geleistete Beihilfe zur Steuerhinterziehung von Bürgerinnen und Bürgern fremder Demokratien nicht herunterspielen und gleichzeitig erwarten, dass die Schweizerinnen und Schweizer die von ihnen ausgeübte Steuerhinterziehung als ernsthaftes Delikt betrachten. ${ }^{13}$ Daran ändert auch nichts, dass immer wieder betont wird, dass das Bankgeheimnis nicht zum Ziel habe, Steuerflucht aus anderen Ländern zu fördern. ${ }^{14}$ Solange die Unterscheidung zwischen Steuerhinterziehung und Steuerbetrug in der heutigen Form aufrechterhalten wird, ist diese Behauptung nicht glaubwürdig und ihre stete Wiederholung durchaus geeignet, zur Steuerhinterziehung im eigenen Land zu ermuntern.

Nun gibt es leider keine Möglichkeit zu überprüfen, welcher dieser beiden Faktoren (neben anderen) für den Rückgang der Steuermoral in welchem Ausmaß verantwortlich ist; Umfragedaten, mit denen man dies untersuchen könnte, liegen nicht vor. Approximativ erfassen kann man jedoch die Intensität der öffentlichen Diskussion zu diesen beiden Themen. So wurde von den Neuen Zürcher Zeitung im Zeitraum von 1993 bis 1997 in insgesamt 173 Artikeln das Problem des Bankgeheimnisses thematisiert, aber nur in 57 Artikeln die Steueramnestie. Dies lässt zumindest vermuten, dass die Diskussion um letztere für die Veränderung in der Einstellung der Bevölkerung weniger wichtig war als die Diskussion um das Bankgeheimnis.

Eine tiefe Steuermoral sollte - ceteris paribus - dazu führen, dass entsprechend viel Steuern hinterzogen werden. Institutionelle Faktoren wie die direkten Volksrechte könnten dem jedoch entgegenwirken. Eine geringe Steuermoral muss deshalb nicht notwendigerweise mit hohen Hinterziehungsraten

11. Nach einer Umfrage der Bankiervereinigung im Jahr 2001 wollen zwar 80 Prozent der Schweizerinnen und Schweizer das Bankgeheimnis beibehalten, aber 67 Prozent fordern seine Aufhebung bei Verdacht auf Steuerhinterziehung. (Siehe: Bankgeheimnis bei Steuerhinterziehung unpopulär; Neue Zürcher Zeitung Nr. 139 vom 19. Juni 2001, S. 21.) - Zur jüngeren Diskussion um das Bankgeheimnis siehe z.B. Bär (2004) und die sich an das Erscheinen dieses Buchs anschließende Diskussion.

12. Zum "Angriff“ auf das Bankgeheimnis siehe z.B.: Angriff auf das Bankgeheimnis abgewehrt, Neue Zürcher Zeitung Nr. 88 vom 13. April 2000, S. 21; Herausgeforderte Schweizer Banken, Neue Zürcher Zeitung Nr. 2 vom 4. Januar 2003, S. 17; Verschnaufpause für das Bankgeheimnis, Neue Zürcher Zeitung Nr. 114 vom 18. Mai 2004, S. 19.

13. Siehe z.B. Das Bankgeheimnis - abschaffen oder stärken?, Neue Zürcher Zeitung Nr. 242 vom 18. Oktober 1999; Blankart, Die Berechtigung des Bankkundengeheimnisses, Neue Zürcher Zeitung Nr. 277 vom 27. November 1999, S. 29.

14. Siehe z.B. Kummer, Die Privatsphäre als Wettbewerbsfaktor, Neue Zürcher Zeitung Nr. 271 vom 20. November 2000, S. 18. 


\section{Direkte Demokratie, Steuermoral und Steuerhinterziehung}

zusammenfallen. Die entsprechenden Ergebnisse für die Schweiz sind widersprüchlich. Es gibt Indizien dafür, dass die Steuerhinterziehung (auch verglichen mit anderen Staaten) recht hoch ist. So wird nach Frey und Feld (2002, S. 36) ein Viertel aller Einkommensteuern hinterzogen. Andererseits deuten international vergleichende Untersuchungen zur Schattenwirtschaft auf eine eher geringe Steuerhinterziehung hin. ${ }^{15}$

Dass andererseits das Vertrauen in das Rechts- und Gerichtswesen nicht allzu stark ausgeprägt ist, hat mit der Geschichte der Schweiz und ihren politischen Strukturen zu tun. In der Schweiz dominierte mit der Ablösung der liberalen durch die radikalen Regierungen in den dreißiger und vierziger Jahren des 19. Jahrhunderts das demokratische gegenüber dem liberalen Prinzip: ${ }^{16}$ Die „freien“ Schweizer waren gewillt, ihre politischen Angelegenheiten selbst zu regeln. Dies bedeutete nicht notwendigerweise die Einführung der direkten Demokratie: Um 1840 gab es zwar in sieben Kantonen eine Landsgemeinde und in sechs Kantonen eine halbdirekte Demokratie, aber 11 Kantone hatten eine rein repräsentative Demokratie, während Neuenburg eine konstitutionelle Monarchie war. ${ }^{17}$ Auch die neue Bundesverfassung des Jahres 1848 hatte nur wenige Elemente der direkten Demokratie; die wichtigsten heute auf eidgenössischer Ebene bestehenden Volksrechte, das fakultative Gesetzesreferendum und die Volksinitiative auf Teilrevision der Verfassung, wurden erst 1874 (im Rahmen der Totalrevision) bzw. 1891 in die Verfassung aufgenommen. Neben diesen demokratischen Rechten hatte der Rechtsstaat eine geringere Bedeutung: Insbesondere dort, wo eine Landsgemeinde existierte, d.h. wo jeder (männliche) Bürger seine Anliegen direkt in den politischen Prozess einbringen konnte, machte es wenig Sinn, gegen politische Entscheidungen bei Gericht klagen zu wollen. ${ }^{18}$ Diese Überzeugung ist bis heute bei der schweizerischen Bevölkerung weit verbreitet; sie zeigt sich z.B. im Widerstand gegen die Einführung einer Verfassungsgerichtsbarkeit auf Bundesebene.

Die Tatsache, dass die Steuermoral in der Schweiz im internationalen Vergleich relativ gering und auch das Vertrauen in das Rechtssystem zumindest nicht sehr stark ausgeprägt ist, ist für sich selbst betrachtet noch wenig bedeutsam. Letztlich entscheidend ist, wieweit sich die Steuerzahler an die Steuergesetze halten. Insofern ist die Beziehung zwischen Steuerhinterziehung und direkter Demokratie sehr viel relevanter als diejenige zwischen direkter Demokratie und Steuermoral. Zudem könnte es trotz allem sein, dass die direkte Demokratie positiv auf die Steuermoral wirkt: Ohne direkte Volksrechte könnte

15. Siehe Weck (1983) oder Schneider (2005).

16. Siehe hierzu Andrey (1983, S. 267) sowie Schaffner (1998). Zum Spannungsverhältnis zwischen liberalem und demokratischem Prinzip siehe Kirchgässner (2004).

17. Eine Landsgemeinde hatten AI, AR, GL, NW, OW, UR und SZ, halbdirekte Demokratien waren $\mathrm{BL}, \mathrm{GR}, \mathrm{LU}$, SG, VS und ZG, während AG, BE, BS, FR, GE, SH, SO, TG, TI, VD und ZH repräsentative Demokratien hatten. Siehe Andrey (1983, S. 267).

18. Dementsprechend entwickelten sich in der Schweiz auch das Verwaltungsrecht und die Verwaltungsgerichtsbarkeit erst später als in Deutschland. Zur Entwicklung in Deutschland siehe Keller (1998). 


\section{Gebhard Kirchgässner}

sie in der Schweiz ja noch viel geringer sein. Im Folgenden muss es daher darum gehen, zu untersuchen, inwieweit die direkte Demokratie nicht nur auf die Steuermoral, sondern vor allem auf die Befolgung der Steuergesetze bzw., das Komplement dazu, die Steuerhinterziehung, Einfluss hat. Dies kann freilich nicht dadurch erfasst werden, dass die Situation der Schweiz mit derjenigen in anderen Staaten verglichen wird, da eine Stichprobe von „Eins“ keine verallgemeinerungsfähigen Aussagen zulässt. Für eine international vergleichende Studie ist zudem die Variation in den direkten Volksrechten zu gering; sieht man von den Ebenen der Bundesstaaten und der lokalen Gebietskörperschaften in den Vereinigten Staaten ab, dann gibt es außerhalb der Schweiz kaum direkte Rechte, mit denen die Bürgerinnen und Bürger Einfluss auf die Besteuerung nehmen können und die damit auch Auswirkungen auf die Steuermoral haben können.

Es besteht jedoch die Möglichkeit, diese Fragestellung innerhalb der Schweiz aufzugreifen. Die direkten Volksrechte sind in den einzelnen Kantonen und Gemeinden sehr unterschiedlich ausgeprägt. Dies gilt insbesondere auch für jene Instrumente, mit denen die Stimmbürger auf die öffentlichen Finanzen Einfluss nehmen können. Die Kantone und Gemeinden der Schweiz eignen sich daher gleichsam wie ein Labor zur Untersuchung der Auswirkungen der direkten Volksrechte nicht nur auf die öffentlichen Finanzen, ${ }^{19}$ sondern auch auf Steuermoral und Steuerhinterziehung. ${ }^{20}$ Im Folgenden sollen daher die Ergebnisse von Untersuchungen dargestellt werden, die sich auf die Ebene der Kantone beziehen. Davor soll jedoch erläutert werden, wie die Stimmbürgerinnen und Stimmbürger in der direkten Demokratie der Schweiz Einfluss auf die Besteuerung nehmen können. Nur wenn dies möglich ist, kann man erwarten, dass die direkte Demokratie die Steuermoral und damit das Ausmaß der Steuerhinterziehung negativ beeinflusst.

\section{Einflussmöglichkeiten der Stimmbürger auf die Besteuerung}

„Direkte Demokratie“ wird hier (und im Folgenden) verstanden als die Möglichkeit der Stimmbürgerinnen und Stimmbürger, über Sachfragen direkt entscheiden zu können. Sie hat somit nichts mit der Direktwahl bestimmter Personen zu tun, die gelegentlich (in Deutschland) damit verwechselt wird. Es handelt sich zweitens um den Bürgerinnen und Bürgern aufgrund der Verfassung oder gesetzlicher Bestimmungen zustehende Rechte, und nicht um Plebiszite, die ihnen von Zeit zu Zeit gnädigerweise von ihrer Obrigkeit gewährt

19. Siehe hierzu z.B. Feld und Kirchgässner (2001, 2004, 2005).

20. Ähnliches gilt für die Bundesstaaten und die lokalen Gebietskörperschaften in den Vereinigten Staaten. Siehe hierzu z.B. Matsusaka (2004). 


\section{Direkte Demokratie, Steuermoral und Steuerhinterziehung}

werden. ${ }^{21}$ Dabei kennt die direkte Demokratie der Schweiz mit der Initiative ein "Gaspedal" und mit dem Referendum eine „Bremse“. ${ }^{22}$ Mit der Initiative können zusätzliche Vorschläge in den politischen Prozess eingebracht werden, welche die gewählten Repräsentanten nicht von sich aus einbringen (bzw. zumindest bisher nicht eingebracht haben). Das Referendum dient da$\mathrm{zu}$, die Umsetzung von Vorschlägen der Repräsentanten, die nicht dem Willen der Stimmbürger entsprechen, zu verhindern. Beide Instrumente helfen somit den Bürgerinnen und Bürgern, sich im Zweifelsfall gegen die Repräsentanten durchzusetzen. Dabei spielt in Steuerfragen die Initiative freilich in der Schweiz (im Gegensatz zu den Vereinigten Staaten) eine eher untergeordnete Rolle.

Eine Initiative (bzw. im bundesrepublikanischen Sprachgebrauch ein Volksbegehren mit anschließendem Volksentscheid) kann sich auf die Verfassung oder ein Gesetz beziehen; daneben gibt es noch die allgemeine Initiative, die es dem Gesetzgeber überlässt, ob er dem Anliegen auf der Gesetzesebene oder auf der Verfassungsebene Rechnung trägt. Auf Bundesebene gibt es seit dem Beginn des Bundesstaats im Jahr 1848 die Initiative auf Totalrevision der Verfassung, die freilich noch niemals in Anspruch genommen wurde. Seit dem Jahr 1891 gibt es daneben die Initiative auf Teilrevision der Verfassung. Zu ihrem Zustandekommen waren zunächst 50.000 und sind seit dem Jahr 1977100.000 Unterschriften erforderlich, die in 18 Monaten gesammelt werden müssen. Dieses Instrument ist seit 1981 159-mal ergriffen worden. Es war jedoch nur in 14 Fällen erfolgreich; in sechs weiteren Fällen wurde der vom Parlament vorgelegte Gegenvorschlag angenommen. Damit sie erfolgreich ist, müssen der Initiative Volk und Stände zustimmen, d.h. es bedarf der Zustimmung der Mehrheit der Abstimmenden sowie auch der Mehrheit der Kantone. Das seit dem Jahr 2003 bestehende Recht zur allgemeinen Initiative wurde bisher noch nicht in Anspruch genommen.

Auf der kantonalen Ebene gibt es überall die Initiative auf Teilrevision der Verfassung. Daneben ist es in vielen Kantonen auch möglich, Gesetzesinitiativen zu ergreifen. Die Zahl der Unterschriften, die hierzu erforderlich ist, ist sehr unterschiedlich. In den Landsgemeindekantonen Appenzell, Innerrhoden und Glarus genügt eine einzelne Stimme, im Kanton Wallis sind mit 12.000 Unterschriften für das Zustandekommen einer Verfassungsinitiative dagegen etwa 7 Prozent der Stimmberechtigten erforderlich, im Kanton Neuenburg mit 6.000 Unterschriften für das Zustandekommen einer Gesetzesinitiative 5,84 Prozent. Viele Kantone kennen keine Frist, in der diese Unterschriften gesammelt werden müssen; die kürzeste Frist beträgt 60 Tage für die Gesetzesinitiative im Kanton Tessin. ${ }^{23}$

21. Zur Systematik der verschiedenen Arten von Volksabstimmungen siehe Frey und Kirchgässner (2002, S. 67ff.).

22. Siehe hierzu Eichenberger (1999). Zur direkten Demokratie der Schweiz siehe auch Kirchgässner, Feld und Savioz (1999).

23. Die Werte beziehen sich auf das Jahr 1992; siehe hierzu Stutzer (2003, S. 217). 


\section{Gebhard Kirchgässner}

Mit Hilfe einer Initiative kann auf die Besteuerung in zweifacher Hinsicht Einfluss genommen werden. Zum einen können Steuersätze (oder auch Steuertarife) direkt verändert werden. So sind z.B. die Maximalsätze der Mehrwertsteuer (Art. 130; Übergangsartikel zu Art. 67) in der Bundesverfassung festgeschrieben. Sie könnten durch eine Initiative verändert werden. Aber auch die Sätze der Mineralölsteuer, die im Anhang zum Mineralölsteuergesetz (SR 641.61) festgelegt sind, könnten durch eine Verfassungsinitiative verändert werden, wodurch diese Sätze dann in der Verfassung festgelegt würden. Zweitens können über Initiativen neue Ausgabenverpflichtungen geschaffen werden, was in der Regel zu höheren Steuerlasten führt. Es kann aber genauso versucht werden, durch Initiativen bestimmte Ausgabenprojekte zu verhindern oder Ausgaben deutlich zu reduzieren, wodurch Raum für Steuersenkungen geschaffen würde. So wurde am 6. Juni 1993 über die Initiative „für eine Schweiz ohne neue Kampfflugzeuge" abgestimmt, die die Beschaffung neuer Abfangjäger (F/A-18) verhindern sollte. Sie ist freilich mit einer Zustimmung von nur 42,8 Prozent gescheitert. Am 24. September 1992 wurde eine Initiative „für weniger Militärausgaben und mehr Friedenspolitik" eingereicht, die eine Halbierung der Militärausgaben zum Ziel hatte. Sie wurde jedoch vom Parlament am 20. Juni 1995 wegen der Verletzung des Grundsatzes der „Einheit der Materie“ für ungültig erklärt (und kam deshalb gar nicht zur Abstimmung).

Diese Beispiele zeigen, dass die Initiative zwar zur Begrenzung der Steuerlast eingesetzt werden kann, dass sie aber diesbezüglich eher eine Krücke ist, die man benutzt, wenn kein geeigneteres Instrument zur Verfügung steht. Das eigentliche Instrument zur Begrenzung der Steuerlast ist das Referendum. Auf Bundesebene gibt es das obligatorische Referendum für Änderungen der Verfassung sowie seit 1874 das fakultative Gesetzesreferendum. Wie bei der Initiative bedarf es beim obligatorischen Referendum des "doppelten Mehrs" der Abstimmenden und der Kantone, während beim fakultativen Referendum das (einfache) Volksmehr genügt. Damit ein fakultatives Referendum zustande kommt, müssen innerhalb von 6 Wochen mindestens 50.000 Unterschriften gesammelt werden. ${ }^{24}$ Das obligatorische Referendum wurde bisher 205mal durchgeführt, wobei Volk und Stände der Vorlage des Parlaments in 155 Fällen zustimmten. Das fakultative Referendum, welches bisher 152-mal ergriffen wurde, führte dagegen in 73 Fällen und damit in knapp 50 Prozent aller Fälle zur Ablehnung der Gesetzesvorlage.

Alle Kantone kennen das obligatorische Referendum auf Teil- und Totalrevision der Verfassung. Die Bereiche, die sonst dem obligatorischen oder fakultativen Referendum unterstehen, sind dagegen sehr unterschiedlich. Dies gilt neben dem Gesetzesreferendum auch für das Finanzreferendum. Mit ihm

24. Bis 1977 waren 30.000 Unterschriften erforderlich. Daneben gibt es seit 1921 das Staatsvertragsreferendum, welches 1977 obligatorisch wurde, sowie seit 1949 das obligatorische Referendum über dringlich erklärte Bundesgesetze, die keine Verfassungsgrundlage haben (Art. 140). Auf diese Instrumente, die für Fragen der Besteuerung kaum relevant sind, soll hier nicht eingegangen werden. 


\section{Direkte Demokratie, Steuermoral und Steuerhinterziehung}

kann über Ausgaben entschieden werden, die keiner zusätzlichen gesetzlichen Grundlage bedürfen. Mit Ausnahme des Kantons Waadt kennen heute alle Kantone zumindest eine der möglichen Formen des fakultativen Finanzreferendums. In 12 Kantonen sind z.B. ordentliche Ausgaben und in 10 Kantonen außerordentliche Ausgaben, die einen bestimmten Betrag überschreiten, dem obligatorischen Referendum unterstellt. Bezüglich der Steuereinnahmen gibt es ein ordentliches obligatorisches Finanzreferendum dagegen heute nur in den Kantonen Bern und Uri. ${ }^{25}$

Das obligatorische Referendum gilt zunächst für die Finanzordnung des Bundes. Sie, und damit die Berechtigung zur Erhebung der direkten Bundessteuer und der Mehrwertsteuer, ist gemäß dem Übergangsartikel zu Artikel 128 der Bundesverfassung zeitlich befristet, derzeit bis zum Jahr 2020. Damit sie verlängert werden kann, bedarf es jeweils einer Verfassungsänderung und damit eines obligatorischen Referendums. Die Verlängerung wurde von den Stimmbürgern in der Vergangenheit mehrfach erst im zweiten Anlauf genehmigt, nachdem der erste Versuch, bei welchem gleichzeitig die Befristung aufgehoben werden sollte, gescheitert war. ${ }^{26}$

Das obligatorische Referendum wird auch dann wirksam, wenn Steuersätze in der Verfassung festgelegt sind. Dies gilt, wie bereits ausgeführt wurde, auf der Bundesebene z.B. für die Mehrwertsteuer. Auch deren Einführung unterlag dem obligatorischen Referendum. Es bedurfte mehrerer Anläufe, bis die Stimmbürger damit einverstanden waren. Der Grund dafür war, dass das Parlament bei den ersten Versuchen diese Einführung mit einer Erhöhung des Steuersatzes verknüpfen wollte. Erst als am 28. November 1993 die Einführung der Mehrwertsteuer und die Erhöhung des Steuersatzes getrennt vorgelegt wurden, stimmte das Volk beidem zu. ${ }^{27}$ Es hat sich somit mehrfach erfolgreich gegen Steuererhöhungen gewehrt.

Das fakultative Referendum kann immer dann (indirekt) zur Senkung der Steuerlast beitragen, wenn Gesetze beschlossen werden sollen, die erhebliche finanzielle Konsequenzen haben. Wie in Kirchgässner und Schulz (2005) gezeigt wurde, ist die Zahl der Nein-Stimmen bei einem Referendum umso größer, je größer der zur Diskussion stehende Betrag ist. Dadurch werden die Chancen, dass die Vorlage durchgeht, erheblich beeinträchtigt. Dem dürfte z.B. am

25. Siehe hierzu Trechsel und Serdült (1999, S. 37ff.).

26. Dies war z.B. zu Beginn der siebziger Jahre der Fall. Die erste Vorlage wurde am 15. November 1970 abgelehnt, die zweite am 6. Juni 1971 angenommen. Es wiederholte sich Ende der siebziger Jahre. Die erste Vorlage wurde am 20. Mai 1979 abgelehnt, die zweite am 29. November 1981 angenommen.

27. Abgelehnt wurde die Einführung der Mehrwertsteuer am 12. Juni 1977 und am 20. Mai 1979. Der Satz der bis 1993 geltenden Warenumsatzsteuer betrug 6,2 Prozent. Zusammen mit dem Wechsel zur Mehrwertsteuer (Warenumsatzsteuer mit Vorsteuerabzug) wurde im November 1993 zum einen eine Erhöhung dieses Satzes auf 6,5 Prozent und zweitens die Option auf eine weitere Erhöhung um einen Prozentpunkt vorgeschlagen. Letztere sollte ausschließlich der Alters- und Hinterlassenenversicherung zugute kommen. Alle drei Vorlagen wurden gutgeheißen. 


\section{Gebhard Kirchgässner}

6. Dezember 1987 das geplante neue Bundesgesetz über die Krankenversicherung zum Opfer gefallen sein.

Neben dem Gesetzesreferendum spielt auf der Kantonsebene das bereits erwähnte Finanzreferendum eine wesentliche Rolle. Gerade auf dieser und auf der Gemeindeebene gibt es viele Ausgabenprojekte, die keiner neuen gesetzlichen Grundlage bedürfen und die deshalb nicht dem Gesetzesreferendum unterliegen. So stand z.B. im Kanton St. Gallen das Projekt der Renovierung und Erweiterung der Universität am 5. Juni 2005 zur Abstimmung. Die (vom Stimmbürger bewilligte) Projektsumme von 83 Millionen Franken überstieg nicht nur die Schwelle des fakultativen Referendums, die für einmalige Ausgaben bei 3 Millionen Franken liegt, sondern auch jene des obligatorischen, die bei 15 Millionen Franken liegt.

Referenden können jedoch nicht nur dazu verwendet werden, zusätzliche Ausgaben zu vermeiden, sondern auch, Steuergeschenke zu verhindern. So wurde am 16. Mai 2004 das „Steuerpaket" wohl vor allem deshalb abgelehnt, weil es - hauptsächlich auf Kosten der Kantone - massive Steuergeschenke für die Besitzer von Eigenheimen und Eigentumswohnungen vorsah. Dagegen hatten (zum ersten Mal in der Geschichte der Eidgenossenschaft) die Kantone das Referendum ergriffen, und die Vorlage wurde mit 65,9 Prozent sehr deutlich verworfen.

Im Unterschied zu den Vereinigten Staaten ist es somit weniger die Initiative als vielmehr das Referendum, mit dessen Hilfe sich die Bürgerinnen und Bürger gegen eine übermäßige Belastung mit Steuern wehren können. Außerdem haben sie durch das Gesetzes- und das Finanzreferendum deutlich mehr Möglichkeiten, auf die Verwendung ihrer Steuergelder Einfluss zu nehmen, als dies in einem rein repräsentativen System der Fall wäre. Dadurch sollte ihre Bereitschaft, Steuern zu zahlen, wachsen und auch ihre Steuermoral positiv beeinflusst werden. Ob dies tatsächlich der Fall ist, ist jedoch eine empirische Frage. Darauf soll in den folgenden Abschnitten eingegangen werden.

\section{Direkte Volksrechte und Steuermoral}

Die Auswirkungen der direkten Volksrechte sowie des Vertrauens auf die Steuermoral wurden in der bereits erwähnten Arbeit von Torgler (2005) untersucht. Er verwendet dafür die oben beschriebenen Daten des ISSP, wobei ihm für die Schweiz für seine wichtigste Schätzung 1068 Beobachtungen zur Verfügung stehen, die im Jahr 1998 erhoben wurden. Abhängige Variablen sind die kodierten Antworten auf die Frage nach der Steuermoral. Mit Hilfe des geordneten Probit-Verfahrens erhielt er folgende Schätzung: ${ }^{28}$

28. Die Zahlen in Klammern sind die Absolutbeträge der z-Werte der geschätzten Parameter. Ähnliche Ergebnisse finden sich in Torgler und Schneider (2004), die mit Daten des WVS gearbeitet haben. Mit beiden Datensätzen ergibt sich ein signifikanter Einfluss der direkten Demokratie auf die Steuermoral. Im Gegensatz zu hier ist dort freilich die Strafsteuer hoch signifikant. 


\section{Direkte Demokratie, Steuermoral und Steuerhinterziehung}

$$
\begin{aligned}
& \mathrm{SM}=-0,00004 \mathrm{P}-0,001 \mathrm{SST}-0,010 \mathrm{TR}+0,104 \mathrm{DDI}+0,093 \mathrm{VER} \\
& \begin{array}{lllll}
(0,05) \quad(0,66) \quad(0,77) \quad(3,41) \quad(2,94) & 0
\end{array} \\
& +0,085 \mathrm{CA}+0,021 \mathrm{INC}+\cdots+\varepsilon \text {. } \\
& (4,75) \quad(0,96)
\end{aligned}
$$

Dabei sind:

SM die Steuermoral,

P die Aufdeckungswahrscheinlichkeit, approximiert durch die Zahl der Steuerbeamten pro 100 Einwohner im Kanton,

SST die Höhe der Strafsteuer, approximiert durch das Verhältnis der üblichen gesetzlichen Strafsteuer in einem Kanton (in Prozent),

TR der persönliche Einkommensteuersatz (in Prozent),

INC das Einkommen (in 1000 Franken),

DDI der Index für das Ausmaß der direkten Volksrechte im Kanton,

VER das Vertrauen in das Rechts- und Justizwesen,

CA die Intensität des Kirchenbesuchs, die ebenfalls im ISSP Survey abgefragt wurde.

Der Index für das Ausmaß der direkten Volksrechte wurde von Stutzer (1999) entwickelt und seitdem in einer Fülle von Arbeiten eingesetzt. ${ }^{29}$ Sein Wertebereich geht von 1 (geringste Volksrechte) bis 6 (umfassendste Volksrechte). Er vereint vier Teilindizes: (i) Verfassungsinitiative, (ii) Gesetzesinitiative, (iii) Gesetzesreferendum und (iv) Verfassungsreferendum. Daneben wurden weitere Variablen wie Alter, Zivilstand und Beschäftigungsstatus in die Schätzgleichung eingeführt.

Von allen betrachteten Variablen sind nur drei wirklich hoch signifikant, und sie haben alle einen positiven Einfluss auf die Steuermoral: das Ausmaß der direkten Volksrechte, das Vertrauen in das Rechts- und Gerichtswesen sowie die Häufigkeit des Kirchenbesuchs. Dagegen haben diejenigen Variablen, welche in der ökonomischen Analyse der Steuerhinterziehung üblicherweise als die zentralen Variablen angesehen werden sind, die Aufdeckungswahrscheinlichkeit und die Höhe der Strafe, ${ }^{30}$ überhaupt keinen relevanten Einfluss auf die Steuermoral. ${ }^{31}$ Auch der Einfluss der anderen Variablen ist nicht signifikant, wenn auch die Vorzeichen hier plausibel sind: Eine höhere Steuerlast führt in der Tendenz eher dazu, Steuerhinterziehung als Kavaliersdelikt abzutun, und ein höheres Einkommen, welches im Allgemeinen mehr Möglichkeiten zur Steuerhinterziehung bietet, die dann auch zumindest teilweise ausgenützt werden dürften, führt - zumindest entsprechend der Theorie der kognitiven Dissonanz - dazu, dass man dieses Verhalten auch eher zu rechtfertigen

29. Siehe auch die Beschreibung des Index im Anhang von Stutzer (2003).

30. Siehe hierzu z.B. Richter (2005). Zur Einführung in bzw. zur Übersicht über die ökonomische

Theorie der Kriminalität siehe z.B. Eide (1994) sowie Entorf (1996).

31. Zur (traditionellen) ökonomischen Theorie der Steuerhinterziehung siehe z.B. Tanzi (1993). 


\section{Gebhard Kirchgässner}

versucht, indem man die moralische Dimension der Steuerhinterziehung herunterspielt.

Um zu untersuchen, welche der verschiedenen direktdemokratischen Instrumente, die im Index zusammengefasst werden, Einfluss auf die Steuermoral haben, verwendet Torgler (2005) in weiteren Schätzungen die einzelnen Teilindizes. Dabei erhält er für die Koeffizienten der Teilindizes folgende Werte:
(i) Verfassungsinitiative 0,051
(ii) Gesetzesinitiative 0,064
(iii) Gesetzesreferendum 0,088
(iv) Finanzreferendum 0,090

Der Koeffizient für die Verfassungsinitiative ist auf dem 10-Prozent-Niveau, derjenige für die Gesetzesinitiative auf dem 5-Prozent-Niveau, und die Koeffizienten für die beiden Referenden sind auf dem 1-Prozent-Niveau signifikant von Null verschieden. Dies zeigt, dass ein direktdemokratisches Instrument umso eher zur Hebung der Steuermoral beiträgt, je mehr Kontrolle es den Bürgerinnen und Bürgern über das Ausgabenverhalten des Staates bzw. über seine Möglichkeiten, Steuern zu erheben, gibt.

Es stellt sich natürlich die Frage, inwieweit diese Ergebnisse über die Schweiz hinaus verallgemeinert werden können. Bezüglich der direkten Demokratie ist dies fraglich, da keine entsprechenden Untersuchungen für die Vereinigten Staaten vorliegen und neben diesen kein anderer (größerer) Staat auch nur annähernd so stark ausgeprägte direkte Volksrechte auf den mittleren und unteren Ebenen hat. Andererseits gibt es keinen Grund anzunehmen, dass sich Bürgerinnen und Bürger anderer Staaten grundsätzlich anders verhalten sollten. Daher kann die Hypothese, dass eine Stärkung der Volksrechte auch in anderen Staaten eine Erhöhung der Steuermoral bewirken würde, zumindest vorläufig aufrecht erhalten bleiben. Bezüglich des Vertrauens in das Rechtssystem ist die Situation anders. Torgler (2003) hat diese Frage auch in einer internationalen Studie mit Hilfe der WVS-Daten untersucht. Dabei war das Vertrauen in das Rechts- und Gerichtswesen unabhängig vom Schätzverfahren jeweils hoch positiv signifikant, das Einkommen hoch negativ signifikant. Mit Hilfe eines älteren Datensatzes, des Taxpayer Opinion Survey, erhielt er für das Vertrauen das gleiche Ergebnis. ${ }^{32}$ Ähnliche Ergebnisse findet er auch in den Staaten des ehemaligen Ostblocks (2003a), Kanada (2003b) sowie in Indien und Japan (2004). Man kann daher davon ausgehen, dass Vertrauen in die rechtlichen und politischen Institutionen eines Landes die Steuermoral hebt.

32. Dort drehte es sich um das Vertrauen in die öffentlich Beschäftigten („public officials“). Zum Taxpayer Opinion Survey siehe Harris and Associates (1988). Zu einer Übersicht über Arbeiten, die das Thema „Vertrauen“ in Bezug setzen zu Problemen der öffentlichen Finanzen, siehe Slemrod (2002). 


\section{Direkte Demokratie, Steuermoral und Steuerhinterziehung}

Versuche, durch repressive Maßnahmen die Steuermoral zu heben, dürften dagegen zum Scheitern verurteilt sein. Dies bedeutet noch nicht, dass repressive Maßnahmen nicht zu mehr Steuerehrlichkeit führen könnten. Schließlich sind, wie oben ausgeführt wurde, Steuerehrlichkeit und Steuermoral zwei sehr verschiedene Konzepte, die sich gegenseitig verstärken mögen, aber nicht generell parallel verlaufen müssen. Dies ist andererseits nur ein weiterer Hinweis darauf, dass es sehr schwierig ist, mit Hilfe von repressiven Maßnahmen moralische Überzeugungen beeinflussen zu wollen; häufig treten gegenteilige Wirkungen auf. Inwieweit ökonomische Anreize neben institutionellen die Steuerehrlichkeit beeinflussen, ist eine weitere, empirisch zu untersuchende Frage, auf die im Folgenden eingegangen werden soll.

\section{Direkte Demokratie und Steuerhinterziehung}

Je stärker die Stimmbürgerinnen und Stimmbürger bei der Festlegung der Höhe der Steuern und ihrer Verwendung Einfluss nehmen können, desto sicherer können sie sein, dass einerseits nicht zu viele Steuern von ihnen verlangt werden und dass andererseits die erhobenen Steuergelder sinnvoll verwendet werden. Daher ist zu erwarten, dass die Bereitschaft Steuern zu bezahlen (die Steuerhinterziehung), in direkten Demokratien - ceteris paribus - höher (geringer) ist als in rein repräsentativen Systemen. ${ }^{33} \mathrm{Ob}$ dies tatsächlich der Fall ist, haben als erste Weck-Hannemann und Pommerehne (1989) untersucht. Für die 26 schweizerischen Kantone und die beiden Jahre 1970 und 1978 haben sie gefragt, inwieweit die Höhe der hinterzogenen (kantonalen) Steuern in Relation zum „wahren“ Einkommen von einer Reihe ökonomischer Variablen abhängen, insbesondere jenen, die von der ökonomischen Analyse der Kriminalität diskutiert werden, aber auch davon, ob es in dem entsprechenden Kanton direkte Volksrechte in Budgetfragen gibt. ${ }^{34}$ Um dies zu erfassen, konstruierten sie eine Hilfsvariable, die dann den Wert „Eins“ annimmt, wenn die Stimmbürger bzw. Steuerzahler zum einen über den Voranschlag des Budgets und damit über den Umfang und die Struktur der kantonalen Leistungen und zweitens entweder über den Steuerfuß oder über die Festlegung der Neuverschuldung abstimmen können. Andernfalls nimmt diese Variable den Wert „Null“ an.

Für die 26 Kantone und für die beiden Jahre 1970 und 1978, d.h. mit insgesamt 52 Beobachtungen, erhielten sie folgende Schätzung: ${ }^{35}$

33. Siehe hierzu Pommerehne, Hart und Frey (1994) sowie Pommerehne, Hart und Feld (1997).

34. Die Höhe des hinterzogenen Einkommens wird dabei durch einen Vergleich der den Steuerbehörden gemeldeten Haushaltbruttoeinkommen und den kantonalen Volkseinkommen, die unabhängig davon ermittelt werden, erfasst. Siehe Weck-Hannemann und Pommerehne (1989, S. 524f.).

35. Siehe Weck-Hannemann und Pommerehne (1989, S. 547). Dort sind noch weitere Schätzgleichungen angegeben, die jedoch, insbesondere was den Einfluss der direkten Demokratie 


\section{Gebhard Kirchgässner}

$$
\begin{aligned}
& \mathrm{AHY}=0,138-0,282 \mathrm{P}-0,114 \mathrm{SST}+0,823 \mathrm{MTR}-0,264 \mathrm{SFG} \\
& \begin{array}{llll}
(0,01) & (2,42) \quad(0,73) \quad(2,32) \quad(2,32)
\end{array} \\
& -5,880 \ln (\mathrm{Y})-1,215 \mathrm{~A} 65+0,443 \mathrm{NLY}+1,413 \mathrm{IR} \\
& (0,82) \quad(3,19) \quad(2,52) \quad(2,03) \\
& -0,013 \text { DIKP }-5,819 \text { DDD }+\varepsilon \text {, } \\
& (0,80) \quad(3,33) \\
& \mathrm{R}^{2}=0,780, \quad \mathrm{FG}=39,
\end{aligned}
$$

Dabei sind:

AHY der Anteil des hinterzogenen am ,,wahren” Einkommen,

P die Aufdeckungswahrscheinlichkeit, approximiert durch die Zahl der Steuerbeamten pro 100 Einwohner im Kanton,

SST die Höhe der Strafsteuer, approximiert durch das Verhältnis der üblichen gesetzlichen Strafsteuer in einem Kanton (in Prozent),

MTR der maximale Grenzsteuersatz im Kanton (in Prozent),

SFG die Steuerfreigrenze,

$\ln (\mathrm{Y})$ der Logarithmus des durchschnittlichen Pro-Kopf-Einkommen im Kanton,

A65 der Anteil der über 65-jährigen,

NLY der Anteil des Nicht-Lohneinkommens,

IR die Inflationsrate,

DIKP eine Hilfsvariable für die Indexierung der kalten Progression, sowie

DDD die Hilfsvariable für die direkte Demokratie.

Von den Variablen, welche die ökonomische Theorie der Kriminalität als relevant ansieht, hat nur die Aufdeckungswahrscheinlichkeit einen signifikanten Einfluss. Die übrigen Variablen zeigen zunächst, dass zum einen der Anreiz zur Steuerhinterziehung eine Rolle spielt, denn ein höherer Grenzsteuersatz führt nach dieser Schätzung zu mehr Steuerhinterziehung, ${ }^{36}$ dass aber auch Möglichkeiten dazu gegeben sein müssen: Ältere Menschen und Lohneinkommensbezieher haben weniger Möglichkeiten zur Steuerhinterziehung; sie hinterziehen daher - ceteris paribus - auch weniger. Die Ergebnisse zeigen aber auch, dass Fairness-Überlegungen eine Rolle spielen dürften. Je höher die implizite Besteuerung durch die Inflation ist, desto mehr Steuern werden

betrifft, qualitativ das gleiche Ergebnis aufweisen. Die Zahlen in Klammern sind wieder die Absolutbeträge der t-Werte der geschätzten Parameter; FG gibt die Zahl der Freiheitsgrade des t-Tests an. Siehe auch die Ergebnisse in Pommerehne und Weck-Hannemann (1996).

36. Dieses empirische Ergebnis, welches sich mit den allgemeinen Vorstellungen über diesen Sachverhalt deckt, ist deshalb nicht selbstverständlich, weil es im grundlegenden Modell der Steuerhinterziehung, welches von Allingham und Sandmo (1972) entwickelt wurde, von der Risikoaversion der Individuen abhängt, ob eine Erhöhung der Steuerbelastung zu mehr oder zu weniger Steuerhinterziehung führt. 


\section{Direkte Demokratie, Steuermoral und Steuerhinterziehung}

hinterzogen. ${ }^{37}$ Dieser Effekt wird möglicherweise abgeschwächt, wenn der Staat durch eine Indexierung der Steuern zumindest auf jenen Teil des Steueraufkommens verzichtet, den er aufgrund der kalten Progression zusätzlich erhält. ${ }^{38}$ Und schließlich hat die direkte Demokratie einen hoch signifikanten Einfluss: Wenn die Bürgerinnen und Bürger direkt darüber mitentscheiden können, wie viel Steuern sie zahlen und was mit ihrem Steuergeld geschieht, sind sie eher bereit, diese zu zahlen, als wenn diese Entscheidungen allein den Parlamenten überlassen bleiben. Dabei ist der Betrag, um den es hier geht, erheblich, er entspricht etwa 30 Prozent jenes Betrags, welcher dem Fiskus infolge Steuerhinterziehung verloren geht: In Kantonen mit direkter Demokratie in Finanzfragen werden im Durchschnitt etwa 30 Prozent weniger Steuern hinterzogen als in Kantonen, die keine solchen Rechte kennen. ${ }^{39}$ Dies belegt sehr klar, dass direkte Volksrechte in Budgetfragen zu einer höheren Steuerehrlichkeitmoral beitragen.

Wie bedeutend dieser Effekt ist, lässt sich auch daran erkennen, dass man die Aufdeckungswahrscheinlichkeit mindestens verdreifachen müsste, um den gleichen Effekt auf die Steuerehrlichkeitmoral zu erzielen, der sich durch Einführung dieser Rechte ergibt. ${ }^{40}$ Dies ist nicht ohne weiteres möglich. Zudem ist fraglich, ob diese Verdreifachung tatsächlich den gewünschten Effekt hätte. Eine Erhöhung der Aufdeckungswahrscheinlichkeit erfordert mehr Personal und ist deshalb mit Kosten verbunden, die wiederum durch höhere Steuern abgedeckt werden müssten. Dies aber könnte den Anreiz zur Steuerhinterziehung wieder verstärken. Insofern scheint die Stärkung der direkten Volksrechte der mehr versprechende Weg zur Hebung der Steuerehrlichkeitmoral zu sein.

Eine ähnliche Untersuchung, jetzt aber für fünf Jahre aus dem Zeitraum 1970 bis 1995, d.h. mit insgesamt 130 Beobachtungen, haben Frey und Feld (2002) durchgeführt. Zusätzlich zu den üblichen Variablen verwendeten sie Indikatoren für die Behandlung der Steuerzahler durch die Steuerbehörden. Diese wurden durch Umfragen bei den kantonalen Steuerbehörden erhoben. ${ }^{41}$ Dies ergab folgende Schätzung: ${ }^{42}$

37. Entsprechende Evidenz für die Vereinigten Staaten liefern Crane und Nourzad (1986).

38. Der geschätzte Koeffizient hat zwar das damit vereinbare Vorzeichen, ist jedoch nicht signifikant von Null verschieden.

39. Siehe Weck-Hannemann und Pommerehne (1989, S. 546).

40. Ebenda.

41. Ein Problem dieser Variablen ist jedoch, dass - abhängig von den in den einzelnen Kantonen üblichen Verfahren - die meisten Steuerzahler gar nicht mit den kantonalen, sondern ausschließlich mit den kommunalen Steuerbehörden in Kontakt kommen. Andererseits können die kantonalen auf die kommunalen Steuerbehörden einwirken, und „große“ Steuerzahler verhandeln möglicherweise auch in jenen Kantonen direkt mit der kantonalen Behörde, in denen die Betreuung der Steuerzahler im Allgemeinen den Gemeinden obliegt. All dies spricht dafür, dass die geschätzten Wertekoeffizienten in der Tendenz nach unten verzerrt sind.

42. Gleichung (4) in Tabelle 2 auf S. 20 in Frey und Feld (2002). Die Zahlen in Klammern sind wieder die Absolutbeträge der t-Statistiken der geschätzten Parameter. Die Gleichung enthält kein konstantes Glied, da Hilfsvariablen für die fünf Zeitpunkte verwendet wurden. 


\section{Gebhard Kirchgässner}

$$
\begin{aligned}
& \mathrm{AHY}=0,066 \mathrm{P}-0,064 \mathrm{SST}+0,709 \mathrm{MTR}+0,423 \mathrm{Y}-1,038 \mathrm{DIKP} \\
& (2,74) \quad(2,48) \quad(4,92) \quad(2,20) \quad(0,91) \\
& \text {-0, } 002 \text { Pop - 0, } 463 \text { A65 - 0, 678 SSE + 0, 403 SAS - 7, 432 French } \\
& (1,94) \quad(1,95) \quad(2,61) \quad(2,03) \quad(3,10) \\
& -2,291 \mathrm{IdV}-2,908 \text { TypP }-5,725 \mathrm{RespP}-6,673 \text { AutP }+\varepsilon \text {, } \\
& (3,14) \quad(3,49) \quad(3,84) \quad(3,31) \\
& \mathrm{R}^{2}=0,798,
\end{aligned}
$$

Dabei sind:

$\begin{array}{ll}\text { AHY } & \begin{array}{l}\text { der Anteil des hinterzogenen am ,,wahren“ Einkommen, } \\ \text { die Aufdeckungswahrscheinlichkeit, approximiert durch die Zahl } \\ \text { der Steuerbeamten pro 100 Einwohner im Kanton, }\end{array} \\ \text { SST } & \begin{array}{l}\text { die Höhe der Strafsteuer, approximiert durch das Verhältnis } \\ \text { der üblichen gesetzlichen Strafsteuer in einem Kanton (in Prozent) }\end{array} \\ \text { MTR } & \begin{array}{l}\text { der maximale Grenzsteuersatz im Kanton, } \\ \text { das durchschnittliche Pro-Kopf-Einkommen im Kanton in 1.000 SFr, }\end{array} \\ \text { Y } & \text { eine Hilfsvariable für die Indexierung der kalten Progression, } \\ \text { DIKP } & \text { die Bevölkerung (in 1.000) } \\ \text { Pop } & \text { der Anteil der über 65-jährigen (in Prozent), } \\ \text { A65 } & \text { der Anteil der Selbständigen (in Prozent), } \\ \text { SSE } & \text { der Anteil der in der Landwirtschaft beschäftigten Erwerbstätigen } \\ \text { SAS } & \text { (in Prozent), } \\ \text { French die Hilfsvariable für die lateinische Schweiz, } \\ \text { IdV }\end{array} \begin{aligned} & \text { der Index der direkten Volksrechte, } \\ & \text { TypV }\end{aligned}$

Im Gegensatz zu den oben aufgeführten Schätzungen von WeckHannemann und Pommerehne (1989) sind hier die beiden traditionellen Variablen für eine repressive Politik signifikant; die Variable für die Aufdeckungswahrscheinlichkeit jedoch mit falschen Vorzeichen. Hier liegt die Vermutung nahe, dass es sich um Umkehrwahrscheinlichkeit handelt: Je höher die geschätzte Steuerhinterziehung in einem Kanton ist, desto mehr macht es Sinn (und zahlt es sich aus), Steuerkommissare einzustellen. Dass die Strafsteuer die Steuerhinterziehung signifikant reduziert, obwohl sie (nach den Ergebnissen von Torgler, 2005) keinen Einfluss auf die Steuermoral hat, macht wieder den Unterschied zwischen dieser und der Steuerehrlichkeit deutlich. Repressive Maßnahmen können, wie auch andere Studien belegen, in bestimmtem 


\section{Direkte Demokratie, Steuermoral und Steuerhinterziehung}

Ausmaß dazu führen, dass weniger Steuern hinterzogen werden, ${ }^{43}$ aber dies muss nicht bedeuten, dass die Bürgerinnen und Bürger deshalb ihre moralische Einstellung ändern. Und ob dies die kostengünstigste Methode ist, die Steuerhinterziehung zu verringern, ist zumindest nach den Ergebnissen von Kucher und Götte (1998) fraglich.

Der Index der direkten Volksrechte hat einen hoch signifikanten negativen Einfluss auf das Ausmaß der Steuerhinterziehung. Insofern ergibt sich das gleiche Bild wie in den früheren Arbeiten von Weck-Hannemann und Pommerehne (1989) sowie Pommerehne und Weck-Hannemann (1996). Interessant an der Studie von Frey und Feld (2002) sind aber vor allem jene Variablen, welche das Verhalten der Steuerbehörden abbilden. Je härter die Steuerbehörden beim Fehlen einer Steuererklärung vorgehen, desto mehr Steuern werden hinterzogen. Entdecken die Steuerbehörden Fehler in der Steuererklärung, dann führt sowohl ein vorsichtiges (respektvolles) als auch ein strenges Vorgehen zu geringerer Steuerhinterziehung; ersteres, so könnte man vermuten, weil dadurch das Vertrauen der Bürgerinnen und Bürger in die kantonale Steuerverwaltung (und damit ganz allgemein in die öffentlichen Behörden) gestärkt wird, letzteres aus Furcht vor repressiven Maßnahmen. ${ }^{44}$

\section{Die Behandlung durch die Steuerbehörden}

Feld und Frey (2002) haben zudem herausgefunden, dass die Steuerzahler in „direkten Demokratien“ von den Steuerbehörden anders behandelt werden als in rein repräsentativen Systemen. In einer Umfrage versuchten sie zu erkunden, wie die Steuerverwaltungen handeln, falls sie einen Verstoß feststellen. Dabei zeigte sich, dass sie in direkten Demokratien den einzelnen Steuerpflichtigen zwar mit mehr Rücksichtnahme begegnen, indem sie sie eher auf Fehler in ihrer Steuererklärung hinweisen und auch in stärkerem Maße deren Steuererklärung von sich aus korrigieren, wenn dies zu Gunsten der Steuerpflichtigen möglich ist, dass aber dann, wenn Strafen ausgesprochen werden, diese härter ausfallen als in rein repräsentativen Systemen. Feld und Frey (2002) erklären dies damit, dass in direkten Demokratien zwischen den Bürgerinnen und Bürgern und ihren Steuerverwaltungen ein stärkeres Vertrauensverhältnis besteht, weshalb die Steuerbeamten den Steuerpflichtigen zwar weniger

43. Siehe z.B. Ledermann (2003).

44. Beim Einfluss des respektvollen Vorgehens könnte freilich auch Umkehrkausalität vorliegen: Je geringer die Steuerhinterziehung in einem Kanton ist, desto weniger sehen sich die Steuerbehörden zu einem forschen Vorgehen veranlasst. - In einer weiteren Schätzung versuchen Frey und Feld (2002, S. 20, Gleichung (5)) mit Hilfe von Interaktionstermen zu zeigen, dass autoritäres Verhalten der Steuerbehörden vor allem in „repräsentativen Demokratien“ und „respektvolles Verhalten“ eher in direkten Demokratien wirksam ist. Die bisher vorgelegten Ergebnisse sind jedoch nicht so eindeutig, dass man diese Behauptung als (vorläufig) gesichert ansehen könnte. 


\section{Gebhard Kirchgässner}

misstrauisch begegnen, andererseits aber ein festgestellter Versuch einer Steuerhinterziehung als Bruch dieser Vertrauensbeziehung auch stärker geahndet wird.

Das Problem an dieser Untersuchung ist, dass keine Begründung gegeben wird, weshalb dieses Vertrauensverhältnis in einer direkten Demokratie - ceteris paribus - stärker sein sollte als in einem rein repräsentativen System. Ein möglicher indirekter Effekt könnte sich daraus ergeben, dass die Steuerbeamten den Steuerpflichtigen mit um so mehr Vertrauen entgegenkommen, je geringer die Steuerhinterziehung in einem Kanton ist, und diese ist, wie oben gezeigt wurde, in Kantonen mit direkten Volksrechten im Budgetprozess geringer als in den anderen Kantonen. ${ }^{45}$ Dieses größere Vertrauen könnte wiederum dazu führen, dass weniger Steuern hinterzogen werden. Dies aber bedeutet, dass diese Ergebnisse als Stützung der Hypothese betrachtet werden können, dass ein stärkeres Vertrauensverhältnis zwischen Steuerbehörden und Steuerzahlern die Steuerhinterziehung reduziert, auch wenn der Pfad, auf dem dies geschieht, offen bleibt.

Dass andererseits nicht nur das Vertrauen gegenüber den Steuerbehörden, sondern ganz allgemein das Vertrauen in die öffentliche Verwaltung eine Rolle spielt, dafür spricht die bereits erwähnte Untersuchung von Kucher und Götte (1998). Sie zeigen für die Stadt Zürich, dass im Zeitraum von 1964 bis 1996 die Bereitschaft, Steuern zu zahlen, umso höher war, je höher die Zustimmung zu den von der Regierung in den Abstimmungen unterbreiteten Vorlagen war. Das Ausmaß der Zustimmung zu den Vorlagen interpretieren sie als Indikator für das Vertrauen in die Regierung.

\section{Abschließende Bemerkungen}

Zunächst erschien es als Widerspruch: Die Schweiz mit ihrer einzigartigen direkten Demokratie hat im internationalen Vergleich eine sehr geringe Steuermoral, obwohl innerhalb der Schweiz die Steuermoral umso höher ist, je stärker die direkten Volksrechte ausgebaut sind. Dieser scheinbare Widerspruch löst sich auf, wenn man berücksichtigt, dass es ganz andere Faktoren sein können, die zu einer relativ geringen Steuermoral in der Schweiz geführt haben. Dies impliziert, dass die Steuermoral in der Schweiz dann, wenn es keine direkten Volksrechte geben würde, noch sehr viel geringer wäre. Außerdem ist die

45. Man sollte davon ausgehen, dass dieses Vertrauensverhältnis um so stärker ist, je geringer der Abstand zwischen den Steuerpflichtigen und ihrer Gemeinde ist. Dies wird in der Arbeit von Feld und Frey (2002) leider nicht untersucht. Sie verwenden zwar die Größe des Kantons als zusätzliche Variable, aber da die zuständigen Steuerbeamten in den meisten Kantonen bei den Gemeinden angesiedelt sind, sagt die Größe des Kantons kaum etwas über den Abstand zwischen den Steuerpflichtigen und den Steuerbeamten. Insofern ist es auch nicht überraschend, dass die Koeffizienten dieser Variablen in keiner der in dieser Arbeit vorgestellten Schätzgleichungen signifikant von Null verschieden sind. 


\section{Direkte Demokratie, Steuermoral und Steuerhinterziehung}

Höhe der Steuermoral nicht allein dafür ausschlaggebend, wie streng sich die Bürgerinnen und Bürger an die Steuergesetze halten. Dem Einfluss der tiefen Steuermoral kann (und dürfte in der Schweiz vermutlich auch) durch hohes Vertrauen in das politische System entgegengewirkt werden.

Dabei heben genau jene direktdemokratischen Instrumente die Steuermoral, welche nach deutscher Lesart dem Finanzvorbehalt des Parlaments unterliegen und deshalb in den deutschen Länderverfassungen von den - auch sonst schon relativ wenig ausgebauten - direkten Volksrechten ausgenommen sind. Die Erfahrung der Schweiz lehrt, dass die Stimmbürger, wenn sie über solche Rechte verfügen, nicht nur um solide Finanzen bemüht sind, sondern auch eher bereit sind, ihren Beitrag zu den staatlichen Aufgaben zu leisten, da stärker ausgebaute direkte Volksrechte - ceteris paribus - eine höhere Steuermoral und weniger Steuerhinterziehung bewirken. Bezüglich der Nachhaltigkeit der öffentlichen Finanzen scheint der Finanzvorbehalt daher von zwei Seiten her eher unproduktiv zu sein. Dies gilt für die Initiative, stärker aber noch für das Referendum und insbesondere für das Finanzreferendum, welches wegen seiner Konstruktion nur zu niedrigeren, aber nicht zu höheren Ausgaben führen kann.

Angesichts der sehr widersprüchlichen empirischen Ergebnisse bleibt freilich offen, ob die Steuerhinterziehung (und damit auch die Schattenwirtschaft) in der Schweiz - verglichen mit anderen Staaten - tatsächlich gering ist. Und offen bleibt auch, warum die Steuermoral in der Schweiz im Vergleich zu anderen Länder relativ niedrig ist, sowie insbesondere, warum sie seit Beginn der neunziger Jahre so stark gesunken ist. Die oben hierzu geäußerten Vermutungen, dass ein Zusammenhang mit der Verteidigung des Bankgeheimnisses besteht, sind zwar konsistent und klingen plausibel, sollten aber noch genauer belegt werden. Hier ist noch reichlich Raum für weitere Forschungen.

\section{Literaturverzeichnis}

Allingham, M.G. und A. Sandmo (1972), Income Tax Evasion: A Theoretical Analysis, Journal of Public Economics 1, 323-338.

Alm, J., G.H. McClelland und W.D. Schulze (1992), Why Do People Pay Taxes?, Journal of Public Economics 48, 21-38.

Andreoni, J.B., B. Erard und J. Feinstein (1998), Tax Compliance, Journal of Economic Literature 36, 818-860.

Andrey, G. (1983), Auf der Suche nach dem neuen Staat (1798-1848), in: Comité pour une Nouvelle Histoire de la Suisse (Hrsg.), Geschichte der Schweiz - und der Schweizer. Helbing und Lichtenhahn, Basel, 177-287.

Bär, H.J. (2004), Seid umschlungen Millionen. Orell Füssli, Zürich 2004.

Bosco, L. und L. Mittone (1997), Tax Evasion and Moral Constraints: Some Experimental Evidence, Kyklos 50, 297-324.

Crane, S.E. und F. Nourzad (1986), Inflation and Tax Evasion: An Empirical Analysis, Review of Economics and Statistics 68, 217-223. 


\section{Gebhard Kirchgässner}

Dorn, D., J.A.V. Fischer, G. Kirchgässner und A. Sousa-Poza (2006), Is It Culture or Democracy? The Impact of Democracy and Culture on Happiness, erscheint in: Social Indicator Research 51.

Eichenberger, R. (1999), Mit direkter Demokratie zu besserer Wirtschafts- und Finanzpolitik: Theorie und Empirie, in H.H. von Arnim (Hrsg.), Adäquate Institutionen: Voraussetzung für "gute" und bürgernahe Politik? Duncker und Humblot, Berlin, 259-288.

Eide, E. (1994), Economics of Crime: Deterrence and the Rational Offender. North-Holland, Amsterdam 1994.

Entorf, H. (1996), Kriminalität und Ökonomie: Übersicht und neue Evidenz, Zeitschrift für Wirtschafts- und Sozialwissenschaften 116, 417-450.

Feld, L.P. und B.S. Frey (2001), Trust Breeds Trust: How Taxpayers are Treated, Economics of Governance 3, 87-99.

Feld, L.P. und G. Kirchgässner (2001), The Political Economy of Direct Legislation: Direct Democracy in Local and Regional Decision-Making, Economic Policy 33, 329-367.

Feld, L.P. und G. Kirchgässner (2004), On the Effectiveness of Debt Brakes, Ludwig Boltzmann Institut zur Analyse wirtschaftspolitischer Aktivitäten, Forschungsbericht Nr. 2004.28, Dezember 2004.

Feld, L.P. und G. Kirchgässner (2005), Sustainable Fiscal Policy in a Federal System: Switzerland as an Example, in: H. Kriesi, P. Farago, M. Kohli und M. Zarin-Nejadan (Hrsg.), Contemporary Switzerland: Revisiting the Special Case. Palgrave Macmillan, Houndmills, 281-296.

Feld, L.P. und J.-R. Tyran (2002), Tax Evasion and Voting: An Experimental Analysis, Kyklos 55, 197-222.

Frey, B.S. und L.P. Feld (2002), Deterrence and Morale in Taxation: An Empirical Analysis, CESifo Working Paper No. 760, München, August 2002.

Frey, B.S. und G. Kirchgässner (2002), Demokratische Wirtschaftspolitik: Theorie und Anwendung. Vahlen, München 1994, 3. Auflage 2002.

Harris, L. and Associates (1988), 1987 Taxpayer Opinion Survey, Conducted for the U.S. Internal Revenue Service, Internal Revenue Service Document No. 7292, Washington D.C.

Inglehart, R. et al. (2004), Human Beliefs and Values: A Cross-Cultural Sourcebook Based on the 1999-2002 Values Surveys. Siglo XXI Editores, Mexico.

Keller, Chr. (1998), Verwaltungsgerichtsbarkeit, in: A. Erler und E. Kaufmann (Hrsg.), Handwörterbuch der deutschen Rechtsgeschichte, Band 5. Erich Schmidt, Berlin, 879883.

Kirchgässner, G. (2003), Moralische Aspekte der Besteuerung, in: M. Rose (Hrsg.), Integriertes Steuer- und Sozialsystem. Physica, Heidelberg, 215-241.

Kirchgässner, G. (2004), Politische Verfassung, in: N. Goldschmidt und M. Wohlgemuth (Hrsg.), Die Zukunft der Sozialen Marktwirtschaft: Sozialethische und ordnungsökonomische Grundlagen. Mohr Siebeck, Tübingen, 203-239, $264 f$.

Kirchgässner, G. und T. Schulz (2005), Expected Closeness or Mobilisation: Why Do Voters Go to the Polls? Empirical Results for Switzerland, 1981-1999, CESifo Working Paper Nr. 1387, München, Januar 2005.

Kirchgässner, G., L.P. Feld und M.R. Savioz (1999), Die direkte Demokratie: Modern, erfolgreich, entwicklungs- und exportfähig. Helbing und Lichtenhahn/Vahlen, Basel/München.

Kucher, M. und L. Götte (1998), Trust Me: An Empirical Analysis of Taxpayer Honesty, Finanzarchiv 54, 429-444. 


\section{Direkte Demokratie, Steuermoral und Steuerhinterziehung}

Ledermann, L. (2003), The Interplay Between Norms and Enforcement in Tax Compliance, Georg Mason University, Law and Economics Working Paper No. 03-12.

Matsusaka, J.G. (2004), For the Many or the Few: How the Initiative Process Changes American Government. University of Chicago Press, Chicago.

Pommerehne, W.W. und H. Weck-Hannemann (1996), Tax Rates, Tax Administration and Income Tax Evasion in Switzerland, Public Choice 88, 161-170.

Pommerehne, W.W., A. Hart und L.P. Feld (1997), Steuerhinterziehung und ihre Kontrolle in unterschiedlichen politischen Systemen, Homo Oeconomicus 14, 469-478.

Pommerehne, W.W., A. Hart und B.S. Frey (1994), Tax Morale, Tax Evasion and the Choice of Instruments in Different Political Systems, Public Finance 49 (Supplement), 52-69.

Richter, W. (2005), Geplante Steuerhinterziehung und ihre effiziente Bestrafung, Vortrag auf der 48. Arbeitstagung des Finanzwissenschaftlichen Ausschusses des Vereins für Socialpolitik, München, 19. Mai 2005.

Schaffner, M. (1998), „Direkte“ oder „indirekte“ Demokratie: Konflikte und Auseinandersetzungen, 1830-1848, in: A. Ernst, A. Tanner und M. Weishaupt (Hrsg.), Revolution und Innovation: Die konfliktreiche Entstehung des schweizerischen Bundesstaates von 1848. Chronos, Zürich, 271-277.

Schneider, F. (2005), Shadow Economies in 145 Countries All Over the World: Estimation Results Over the Period 1999 to 2003, mimeo, Universität Linz, Juni 2005.

Slemrod, J. (2002), Trust in Public Finance, NBER Working Paper No. 9187, September 2002.

Stutzer, A. (1999), Demokratieindizes für die Kantone der Schweiz, IEW Working Paper Nr. 23, Zürich, Oktober 1999.

Stutzer, A. (2003), Eine ökonomische Analyse menschlichen Wohlbefindens. Shaker, Aachen.

Tanzi, V. (1993), A Primer on Tax Evasion, IMF Staff Papers 40, 807-829.

Torgler, B. (2003), Tax Morale, Rule-Governed Behaviour and Trust, Constitutional Political Economy 14, 119-140.

Torgler, B. (2003a), Tax Morale in Transition Countries, Post-Communist Economies 15, $357-381$.

Torgler, B. (2003b), To Evade Taxes or Not to Evade: That Is the Question, Journal of Socio-Economics 32, 283-302.

Torgler, B. (2004), Tax Morale in Asian Countries, Journal of Asian Economics 15, 237 266.

Torgler, B. (2005), Tax Morale and Direct Democracy, European Journal of Political Economy 21, 525-531.

Torgler, B. und F. Schneider (2004), Does Culture Influence Tax Morale? Evidence from Different European Countries, CREMA Working paper Nr. 2004-17, Basel 2004.

Trechsel, A.H. und U. Serdült (1999), Kaleidoskop Volksrechte. Die Institutionen der direkten Demokratie in den schweizerischen Kantonen (1970-1996). Helbing und Lichtenhahn, Basel et al.

Tretter, B. (1974), Die Steuermentalität: Ein internationaler Vergleich. Duncker und Humblot, Berlin.

Vihanto, M. (2003), Tax Evasion and the Psychology of the Social Contract, Journal of Socio-Economics 32, 111-125.

Weck, H. (1983), Schattenwirtschaft: Eine Möglichkeit zur Einschränkung der öffentlichen Verwaltung?, Lang, Frankfurt. 


\section{Gebhard Kirchgässner}

Weck-Hannemann, H. und W.W. Pommerehne (1989), Einkommensteuerhinterziehung in der Schweiz: Eine empirische Analyse, Schweizerische Zeitschrift für Volkswirtschaft und Statistik 125, 515-556.

Abstract: First it is clarified what "trust" and "tax morale" actually mean and how they are measured. Especially in German-speaking countries, tax morale is often mixed with tax compliance. But the first is a moral conviction while the second denotes actual behaviour, and both do not necessarily need to be congruent. Then it is shown how direct popular rights empower Swiss citizens to influence their tax burden. Finally, we discuss the impact of direct popular rights on tax morale and on tax compliance as well as the impact of the trust between citizens and tax authorities on the latter. It is shown that direct democracy leads to higher tax morale and compliance, even if tax morale is today rather low in Switzerland compared to other countries. 


\section{Direkte Demokratie, Steuermoral und Steuerhinterziehung}

Tabelle A1 Indexwerte der Steuermoral und des Vertrauens

\begin{tabular}{|c|c|c|c|c|c|c|c|c|}
\hline & \multicolumn{4}{|c|}{ WVS-Index } & \multicolumn{4}{|c|}{ ISSP-Index } \\
\hline & \multicolumn{2}{|c|}{ Steuermoral } & \multicolumn{2}{|c|}{ Vertrauen } & \multicolumn{2}{|c|}{ Steuermoral } & \multicolumn{2}{|c|}{ Vertrauen } \\
\hline & Wert & Rang & Wert & Rang & Wert & Rang & Wert & Rang \\
\hline Ägypten & 1,58 & 13 & & & & & & \\
\hline Albanien & 1,88 & 19 & & & & & & \\
\hline Algerien & 2,01 & 27 & & & & & & \\
\hline Argentinien & 1,88 & 20 & & & & & & \\
\hline Armenien & 3,68 & 77 & 2,92 & 44 & & & & \\
\hline Aserbaidschan & 3,62 & 76 & 2,57 & 20 & & & & \\
\hline Australien & 2,16 & 40 & 2,71 & 33 & 1,83 & 5 & 3,53 & 14 \\
\hline Bangladesh & 1,06 & 1 & & & & & & \\
\hline Belgien & 3,61 & 75 & 2,84 & 39 & & & & \\
\hline Bosnien-Herzegowina & 1,76 & 16 & & & & & & \\
\hline Brasilien & 3,59 & 74 & 2,51 & 14 & & & & \\
\hline Bulgarien & 1,99 & 25 & 2,91 & 42 & 1,79 & 3 & 4,74 & 27 \\
\hline Chile & 2,17 & 41 & & & 2,02 & 17 & 4,63 & 26 \\
\hline China & 1,57 & 12 & & & & & & \\
\hline Dänemark & 2,00 & 26 & 2,07 & 1 & 1,86 & 6 & 2,13 & 1 \\
\hline Deutschland (Ost) & 2,37 & 53 & 2,35 & 7 & 2,24 & 25 & 4,29 & 19 \\
\hline Deutschland (West) & & & & & 2,43 & 30 & 3,38 & 9 \\
\hline Dominikanische Republik & 1,95 & 23 & 3,10 & 46 & & & & \\
\hline El Salvador & 1,91 & 21 & 2,66 & 27 & & & & \\
\hline Estland & 3,18 & 70 & 2,80 & 36 & & & & \\
\hline Finnland & 2,55 & 58 & 2,27 & 5 & & & & \\
\hline Frankreich & 3,04 & 67 & 2,68 & 29 & 2,27 & 27 & 5,41 & 32 \\
\hline Georgien & 2,74 & 62 & 2,69 & 31 & & & & \\
\hline Griechenland & 3,16 & 69 & 2,68 & 30 & & & & \\
\hline Großbritannien & 2,43 & 56 & 2,55 & 17 & 2,09 & 20 & 5,21 & 31 \\
\hline Indien & 2,14 & 38 & & & & & & \\
\hline Indonesien & 1,54 & 10 & & & & & & \\
\hline Iran & 1,47 & 7 & & & & & & \\
\hline Irland & 2,29 & 46 & 2,40 & 11 & 2,28 & 28 & 3,44 & 12 \\
\hline Island & 2,23 & 43 & 2,12 & 2 & & & & \\
\hline Israel & & & & & 2,10 & 21 & 2,28 & 3 \\
\hline Italien & 2,39 & 54 & 2,82 & 38 & 2,01 & 15 & 4,77 & 28 \\
\hline Japan & 1,46 & 6 & & & 1,62 & 1 & 3,98 & 16 \\
\hline Jordanien & 1,51 & 8 & & & & & & \\
\hline Kanada & 2,02 & 29 & & & 1,92 & 9 & 4,41 & 22 \\
\hline Kolumbien & 1,92 & 22 & 2,55 & 16 & & & & \\
\hline Kroatien & 2,74 & 63 & 2,76 & 34 & & & & \\
\hline Lettland & 2,36 & 52 & 2,60 & 22 & 2,42 & 29 & 4,91 & 29 \\
\hline Litauen & 3,84 & 78 & 3,03 & 45 & & & & \\
\hline Luxemburg & 3,35 & 71 & 2,41 & 12 & & & & \\
\hline Malta & 1,53 & 9 & 2,66 & 28 & & & & \\
\hline Marokko & 1,25 & 4 & & & & & & \\
\hline Mazedonien & 2,30 & 47 & & & & & & \\
\hline
\end{tabular}




\section{Gebhard Kirchgässner}

Tabelle A1 Indexwerte der Steuermoral und des Vertrauens (Schluss)

\begin{tabular}{|c|c|c|c|c|c|c|c|c|}
\hline & \multicolumn{4}{|c|}{ WVS-Index } & \multicolumn{4}{|c|}{ ISSP-Index } \\
\hline & \multicolumn{2}{|c|}{ Steuermoral } & \multicolumn{2}{|c|}{ Vertrauen } & \multicolumn{2}{|c|}{ Steuermoral } & \multicolumn{2}{|c|}{ Vertrauen } \\
\hline & Wert & Rang & Wert & Rang & Wert & Rang & Wert & Rang \\
\hline Mexiko & 2,31 & 48 & & & & & & \\
\hline Moldawien & 4,18 & 79 & & & & & & \\
\hline Montenegro & 2,55 & 59 & & & & & & \\
\hline Neuseeland & 2,31 & 49 & 2,55 & 18 & 1,97 & 12 & 4,38 & 21 \\
\hline Niederlande & 2,74 & 64 & 2,54 & 15 & 2,17 & 23 & 3,78 & 15 \\
\hline Nigeria & 2,03 & 31 & & & & & & \\
\hline Nordirland & 2,36 & 51 & 2,58 & 21 & 2,16 & 22 & 4,03 & 18 \\
\hline Norwegen & 2,71 & 61 & 2,24 & 4 & 1,98 & 13 & 2,23 & 2 \\
\hline Österreich & 2,10 & 35 & 2,19 & 3 & 2,52 & 31 & 2,62 & 5 \\
\hline Pakistan & 1,19 & 3 & & & & & & \\
\hline Peru & 2,11 & 36 & & & & & & \\
\hline Philippinen & 3,16 & 68 & & & 2,06 & 19 & 2,60 & 4 \\
\hline Polen & 2,14 & 37 & 2,63 & 24 & 1,99 & 14 & 4,29 & 20 \\
\hline Portugal & 2,44 & 57 & 2,76 & 35 & 1,95 & 10 & 4,59 & 25 \\
\hline Puerto Rico & 2,01 & 28 & & & & & & \\
\hline Rumänien & 2,79 & 65 & 2,70 & 32 & & & & \\
\hline Russland & 2,98 & 66 & 2,85 & 40 & 2,78 & 32 & 4,43 & 23 \\
\hline Schweden & 2,42 & 55 & 2,36 & 8 & 1,82 & 4 & 2,84 & 6 \\
\hline Schweiz & 2,65 & 60 & 2,31 & 6 & 2,23 & 24 & 3,43 & 10 \\
\hline Serbien & 2,09 & 34 & & & & & & \\
\hline Singapur & 2,04 & 32 & & & & & & \\
\hline Slowakei & 2,15 & 39 & 2,81 & 37 & 2,02 & 16 & 3,16 & 8 \\
\hline Slowenien & 2,34 & 50 & 2,60 & 23 & 1,89 & 8 & 3,43 & 11 \\
\hline Spanien & 2,25 & 45 & 2,63 & 25 & 1,68 & 2 & 4,02 & 17 \\
\hline Südafrika & 2,23 & 44 & & & & & & \\
\hline Südkorea & 1,59 & 14 & & & & & & \\
\hline Taiwan & 1,96 & 24 & 2,36 & 9 & & & & \\
\hline Tansania & 1,73 & 15 & & & & & & \\
\hline Tschechien & 2,02 & 30 & 2,90 & 41 & 1,96 & 11 & 5,07 & 30 \\
\hline Türkei & 1,18 & 2 & 2,43 & 13 & & & & \\
\hline Uganda & 3,58 & 73 & & & & & & \\
\hline Ukraine & 3,41 & 72 & 2,91 & 43 & & & & \\
\hline Ungarn & 2,09 & 33 & 2,64 & 26 & 2,03 & 18 & 3,48 & 13 \\
\hline Uruguay & 1,76 & 18 & 2,36 & 10 & & & & \\
\hline Venezuela & 1,82 & 18 & & & & & & \\
\hline Vereinigte Staaten & 2,22 & 42 & & & 1,88 & 7 & 4,53 & 24 \\
\hline Vietnam & 1,31 & 5 & & & & & & \\
\hline Weißrussland & 4,22 & 80 & 2,56 & 19 & & & & \\
\hline Zimbabwe & 1,56 & 11 & & & & & & \\
\hline Zypern & & & & & 2,24 & 26 & 2,86 & 7 \\
\hline
\end{tabular}

Im WVS-Index beziehen sich die Werte für die Schweiz und für Australien auf den Durchschnitt der Jahre 1995 bis 1997, da für die in den Jahren 1999 bis 2000 gezogene Stichprobe keine Angaben für diese beiden Länder vorhanden sind. 